\title{
ARTIGOS
}

Recebido em 04.06.2013. Aprovado em 17.09.2013

Avaliado pelo sistema double blind review. Editor Científico: Luiz Carlos Di Serio

http://dx.doi.org/10.1590/S0034-759020140407

\section{COMPARTILHANDO CONHECIMENTO EM PROJETO AUTOMOTIVO: O ECOSPORT DA FORD DO BRASIL}

\author{
Knowledge sharing in an automobile project: Brazil's Ford Ecosport \\ Compartiendo conocimiento en proyecto automotriz: Ford Ecosport de Brasil
}

\section{RESUMO}

A experiência de uma das equipes de desenvolvimento do projeto do novo EcoSport na Ford Motor Camaçari / BA foi investigada quanto ao compartilhamento de conhecimento entre os seus membros. 0 objetivo era avaliar aquela experiência, com vistas ao aprimoramento de futuros trabalhos. 0 foco da investigação foi posicionado em duas questões: a) Como o conhecimento foi compartilhado? e b) Qual a efetividade do compartilhamento de conhecimento? Para obter respostas a essas perguntas, foi realizada uma pesquisa entre os membros da equipe, seguida por uma análise da rede social composta por eles. Os resultados mostraram membros isolados ou com poucos laços entre eles. Também foi revelado que a rede não estava conectada a outros atores importantes da empresa. Os modos de socialização na captação de conhecimentos e de externalização na transmissão de conhecimentos foram aqueles de maior preferência e efetividade. Concluiu-se que um maior equilíbrio no compartilhamento de conhecimento, estimulando os modos de internalização e de combinação, contribuirá para a melhoria do desempenho da equipe.

PALAVRAS-CHAVE | Desenvolvimento de produto, difusão de conhecimento, análise de redes sociais, projeto automotivo, modelo SECl.

\begin{abstract}
The experience lived by a project development team for the new EcoSport at Ford Motor in Camaçari, Bahia was investigated regarding the knowledge sharing between its members. The goal was to evaluate that process, aimed at improving the team's work on new projects. The research focus was placed on two questions: a) what modes of knowledge sharing were preferably practiced. b) What was the effectiveness of these modes on the development process? To answer these questions, we conducted a survey among team's members followed by an analysis of their social network. The research found isolated members or with few ties between them. The network was also isolated in relation to other important actors of the company. The modes of socialization for capturing knowledge and externalization for the transmission of knowledge were the most preferred and effective by the majority of the team members. The authors concluded that it is highly recommended to increase the balance of the knowledge sharing via the stimulation of modes of combination and internalization.
\end{abstract}

renelson.sampaio@fieb.org.br Professor do Programa de PósGraduação Stricto Sensu da Faculdade de Tecnologia SENAI CIMATEC, Salvador, BA - Brasil.

\section{FRANCISCO UCHOA PASSOS}

francisco.uchoa@fieb.org.br Professor do Programa de PósGraduação Stricto Sensu da Faculdade de Tecnologia SENAI CIMATEC, Salvador, BA - Brasil.

\section{VICTOR ASSIS}

vmassis@gmail.com Mestre em Gestão e Tecnologia Industrial pela Faculdade de Tecnologia SENAI CIMATEC, Salvador, $B A-$ Brasil.
KEYWORDS / Product development, diffusion of knowledge, social networks analysis, automobile project, SECI model.

\section{RESUMEN}

Se investigó la experiencia de uno de los equipos de desarrollo del proyecto del nuevo EcoSport en la Ford Motor Camaçari / BA en cuanto al intercambio de conocimiento entre sus miembros. El objetivo era evaluar dicha experiencia, con miras al perfeccionamiento de futuros trabajos. El foco de la investigación se basó en dos cuestiones: a) ¿Cómo se compartió el conocimiento? b) ¿Cuál es la efectividad de compartir conocimiento? Para obtener respuestas para estas preguntas, se realizó una investigación entre los miembros del equipo, seguida de un análisis de la red social compuesta por ellos. Los resultados mostraron miembros aislados o con pocos lazos entre ellos. También fue revelado que la red no estaba conectada con otros actores importantes de la empresa. Los modos de socialización en la captación de conocimientos y de externalización en la transmisión de conocimientos fueran los de mayor preferencia y efectividad. Se concluyó que un mayor equilibrio en el intercambio de conocimiento, estimulando los modos de internalización y de combinación, contribuirá para la mejora del desempeño del equipo.

PALABRAS CLAVE / Desarrollo de producto, difusión de conocimiento, análisis de redes sociales, proyecto automotivo, modelo SECl. 


\section{INTRODUÇÃO}

A empresa industrial tem sentido a crescente necessidade de um maior entendimento dos fluxos de conhecimentos que ocorrem no desenvolvimento de produtos complexos, como é o caso dos produtos automotivos. A gestão do conhecimento vem sendo, assim, reconhecida como um fator relevante no projeto de veículos automotores. A questão prática que se coloca é saber como gerenciar efetivamente o conhecimento empregado naquele ambiente de projeto, disseminando-o, expandindo-o e utilizando-o eficazmente, com vistas a projetos cada vez melhores. Para isso, as formas de compartilhamento do conhecimento deveriam ser entendidas, avaliadas e exploradas de modo adequado.

Infelizmente, as tentativas de avaliar a efetividade do compartilhamento de conhecimento em ambientes como o do desenvolvimento de produtos complexos nem sempre são satisfatórias. A esse respeito, Stefanovitz e Nagano (2009) veem o projeto de um novo produto complexo como uma atividade paradigmática do compartilhamento de conhecimento, o qual ocorre, no entanto, em meio a considerável incerteza quanto ao valor da sua real contribuição para o processo de desenvolvimento do produto (DP). Eles lembram, também, que o compartiIhamento de conhecimento não é uma atividade paralela ao DP, mas, sim, uma parte natural daquele processo, resultante das várias iterações que ocorrem durante a sua execução. Essa característica contribui para dificultar, ainda mais, a explicitação dos fluxos de conhecimentos e a aferição de sua efetividade no ambiente de projeto.

Mesmo diante dessas dificuldades, os autores deste trabalho, seguindo a linha de pesquisa adotada em estudos de caso semelhantes (Sampaio, Moraes, \& Passos, 2013; Sampaio, Rosa, \& Pereira, 2012), decidiram tentar identificar formas de compartilhamento de conhecimentos e aferir sua efetividade no desenvolvimento do projeto do novo modelo EcoSport pela divisão South America Operations da Ford Motor Company do Brasil, em Camaçari, Bahia. O novo EcoSport teve seu projeto desenvolvido entre 2009 e 2011, tendo sido lançado no ano de 2012, com apresentação realizada simultaneamente na Índia e no Brasil.

Em razão da reconhecida complexidade de aferir as formas e a efetividade do compartilhamento de conhecimento em toda a extensão do longo processo de desenvolvimento daquele produto, resolveu-se focalizar apenas o trabalho da equipe responsável pelo projeto Computer Aided Design (CAD) da carroceria do novo automóvel, conhecida, na empresa, como "equipe Body CAD" ou, simplesmente, BCAD, como será referida a partir de agora. O conhecido Modelo SECl, proposto por
Nonaka e Takeuchi (1997), cuja heurística explica a dinâmica de conversões do conhecimento, forneceu elementos para o modelo de investigação, enquanto a metodologia de análise de redes sociais (Wasserman \& Faust, 1999) emprestou o ferramental analítico.

O interesse que motivou a pesquisa empírica relatada neste texto foi saber que modos de conversão de conhecimento estavam ocorrendo prioritariamente no BCAD e como os membros da equipe estavam avaliando os efeitos do compartilhamento de conhecimento. Um fator adicional de estimulo à pesquisa foi a possibilidade de utilizar, mesmo parcialmente, ferramentas analíticas menos subjetivas para aferir aspectos de gestão do conhecimento. Considerou-se que tais ferramentas seriam mais valiosas do que as avaliações meramente qualitativas (Xu \& Bernard, 2011). Os autores deste trabalho entenderam que, identificando-se a natureza das conversões de conhecimento que ocorreram no BCAD durante o desenvolvimento do novo EcoSport e captando-se a percepção das pessoas envolvidas quanto à sua efetividade, um primeiro passo seria dado na direção de, eventualmente, intervir-se no processo de compartilhamento de conhecimento daquela equipe, de modo favorável ao desenvolvimento de produtos vindouros.

Duas premissas deram ensejo à verificação empírica. A primeira delas apoia-se em Nonaka, Toyama e Konno (2000), assumindo que a rede informal de relacionamentos construída no compartilhamento de conhecimentos entre os membros de uma equipe de trabalho entre si, bem como entre estes e os membros de outras equipes da organização, deveria ser fortalecida, pois contribui para a capacitação da equipe. A segunda premissa tem como referência Bhatt (2001) e argumenta que os resultados do compartilhamento de conhecimento são mais efetivos quando há um equilíbrio entre os modos de conversão de conhecimento.

Para a investigação, as duas seguintes questões norteadoras foram colocadas:

a. Como o conhecimento foi compartilhado na equipe do BCAD durante o projeto do novo EcoSport? e

b. Qual a percepção dos membros da equipe sobre a efetividade do compartilhamento de conhecimento?

Essas questões serão respondidas neste texto, que contém a presente seção introdutória, seguida da seção que traz uma rápida revisão da bibliografia que apoiou o estudo. Na seção seguinte, encontram-se os critérios metodológicos da investigação e, em seguida, são apresentados os resultados encontrados. A última seção traz as conclusões do estudo e faz sugestões para a melhoria do processo de compartilhamento de conhecimento nas equipes de projeto investigadas. 


\section{UM BREVE APORTE TEÓRICO}

\section{Modos de conversão do conhecimento}

Nonaka e Takeuchi (1997), com o seu difundido modelo Socialização, Externalização, Combinação e Internalização (SECI), que explica os modos de conversão do conhecimento, já haviam focado a questão do compartilhamento do conhecimento em grandes empresas globais, mas aprofundaram-se, particularmente, nas de origem japonesa. Andreeva e Ikhilchik (2011) fazem uma utilização do modelo SECl em seu país de origem, a Rússia, e mencionam, ainda, os trabalhos de Holden e Glisby (2010) e de Weir e Hutchings (2005), que analisaram a validade do modelo SECI nas culturas chinesa e árabe, levantando vários pontos de alinhamento e também de divergências no que se refere ao compartilhamento de conhecimento em diferentes culturas. Algum tempo depois, Chang, Tsai e Tsai (2011) analisaram como se dá o processo de conversão do conhecimento em 107 empresas de Taiwan, de pequeno e médio portes, todas relativamente intensivas de tecnologia. Inspirando-se nesses estudos, o presente trabalho tem a pretensão de ampliar o quadro, analisando aspectos do modelo $\mathrm{SECl}$ num ambiente de trabalho comum a empresas multinacionais com operações no Brasil. Esses ambientes normalmente se destacam pela heterogeneidade da equipe. No caso particular do projeto do novo EcoSport, tal heterogeneidade atingiu nivel de grande expressão, pois havia diversificação em características culturais, idades, raças, tipo e nível de formação, origem e, naturalmente, atitudes e visões de mundo. Até mesmo os brasileiros da equipe eram oriundos de várias regiões do País e interagiam com americanos, ingleses, indianos, chineses, coreanos, italianos e portugueses. Como eventual contribuição ao conjunto desses estudos, a presente investigação tenta apresentar mais um contraponto ao trabalho original de Nonaka e Takeuchi, ao utilizar o modelo SECl em um ambiente eclético de uma subsidiária de corporação transnacional operando em um país emergente.

$\mathrm{Na}$ tentativa de estabelecer o mencionado contraponto, uma ressalva deve ser feita quanto ao entendimento do que seja "conhecimento". Os autores deste trabalho valeramse das ideias de Xu e Bernard (2011), que se preocuparam com a questão do conhecimento focado apenas no processo de DP, em que é visto como um "insumo com potencial de gerar e modificar produtos". Esse entendimento mais restrito foi considerado mais adequado ao caso específico do desenvolvimento do novo EcoSport.

A partir deste ponto, faz-se uma rápida revisão conceitual sobre a questão da conversão do conhecimento e, a seguir, examinam-se elementos da análise de redes sociais que fundamentaram a avaliação empírica realizada.

Nielsen (2006) considera que as capacidades dinâmicas da empresa intensiva de tecnologia - conceito que expressa 0 ajuste adequado da empresa ao seu ao ambiente de negócio estão fortemente vinculadas a oito processos de conversão do conhecimento, que foram identificados como: criação; aquisição; captura; montagem; compartilhamento; interação; alavancagem; e exploração. Esses processos compõem três capacidades dinâmicas básicas da empresa: (a) desenvolvimento do conhecimento; (b) recombinação do conhecimento; e (c) emprego do conhecimento. Por sua vez, essas capacidades seriam responsáveis pelo estabelecimento dos fluxos (entrada e saída) de conhecimentos e pela manutenção e expansão do estoque de conhecimentos da empresa.

Bhatt (2001) já havia identificado cinco processos de conversão do conhecimento, quais sejam: criação; validação (atualização); apresentação (em mídia); distribuição (estabelecimento de fluxos); e aplicação (emprego em inovações). Ele argumenta que um conveniente equilíbrio entre esses processos de conversão é condição necessária para que a empresa explore, da melhor forma, seus ativos de conhecimento. Um desequilíbrio entre eles, situação em que haveria modos preferenciais de conversão de conhecimento em detrimento de outros, poderia conduzir a empresa em direção a um dos seguintes extremos: (a) excesso de ênfase nas ferramentas tecnológicas de obtenção/tratamento de dados ou (b) excesso de ênfase nos relacionamentos das redes sociais, estabelecidos por pura iniciativa e motivação de pessoas. Um ponto de equilíbrio entre esses extremos se refletiria em um ambiente com dosagem correta de fatores tecnológicos e sociais. Para o adequado balanceamento dos modos de conversão do conhecimento, seriam necessárias intervenções com impactos na cultura, nas tecnologias e nas práticas empresariais. A organização teria que construir um ambiente propício, que estimulasse a aprendizagem pela prática corrente (learning-by-doing).

A espiral do conhecimento, conforme a proposta inicial de Nonaka e Takeuchi (1997), tornou-se um modelo recorrentemente empregado em estudos sobre a dinâmica de conversão do conhecimento, em razão de sua maior simplicidade, pois sintetiza as conversões em apenas quatro modos, concebidos por aqueles autores para explicar a criação de conhecimento novo no ambiente organizacional. 0 modelo está apoiado, em boa medida, nos trabalhos originais de Polanyi (1962) e Polanyi (1967), que considera o conhecimento tácito contido no indivíduo a fonte e origem de todas as outras expressões de conhecimento. Na ilustração da espiral (Figura 1), a socialização é um modo de conversão que pode ser visto como o ponto de partida para o processo completo da criação de co- 
nhecimento, sendo caracterizado pela interação entre os indivíduos, que se comunicam sem necessidade de códigos ou formas explícitas para a mensagem. A externalização é a ação de veicular ideias e conceitos já socializados, fazendo-se uso de uma mídia qualquer, na qual a mensagem será explicitada e codificada. Ao veicular um conhecimento "socializado" utilizando-se uma mídia, o compartilhamento ficaria favorecido, pois tanto os indivíduos que socializaram aquele conhecimento quantos outros indivíduos terão oportunidade de acessá-lo, sempre que dele necessitarem.

A combinação é a ação de reunir conhecimentos explícitos existentes, formando novos pacotes de conhecimento que teriam utilidade em aplicações que precisassem deles. A etapa que fecha o ciclo de conversões de conhecimento é a internalização, em que o "aprender fazendo" transforma o conhecimento explicitado nas duas etapas anteriores em conhecimento tácito novamente, iniciando-se, assim, um novo ciclo de conversões, o que faz expandir a espiral de conversões, resultando na ampliação do compartilhamento de conhecimento, tanto em profundidade de conteúdo quanto em número de indivíduos e grupos alcançados. A cada iteração da espiral, o conhecimento consolida-se, amplia-se e dissemina-se entre os indivíduos, grupos e organizações, e novos conhecimentos poderão ser criados.

\section{Figura 1. Espiral do conhecimento}

\section{Quatro modos de conversão de conhecimento:}

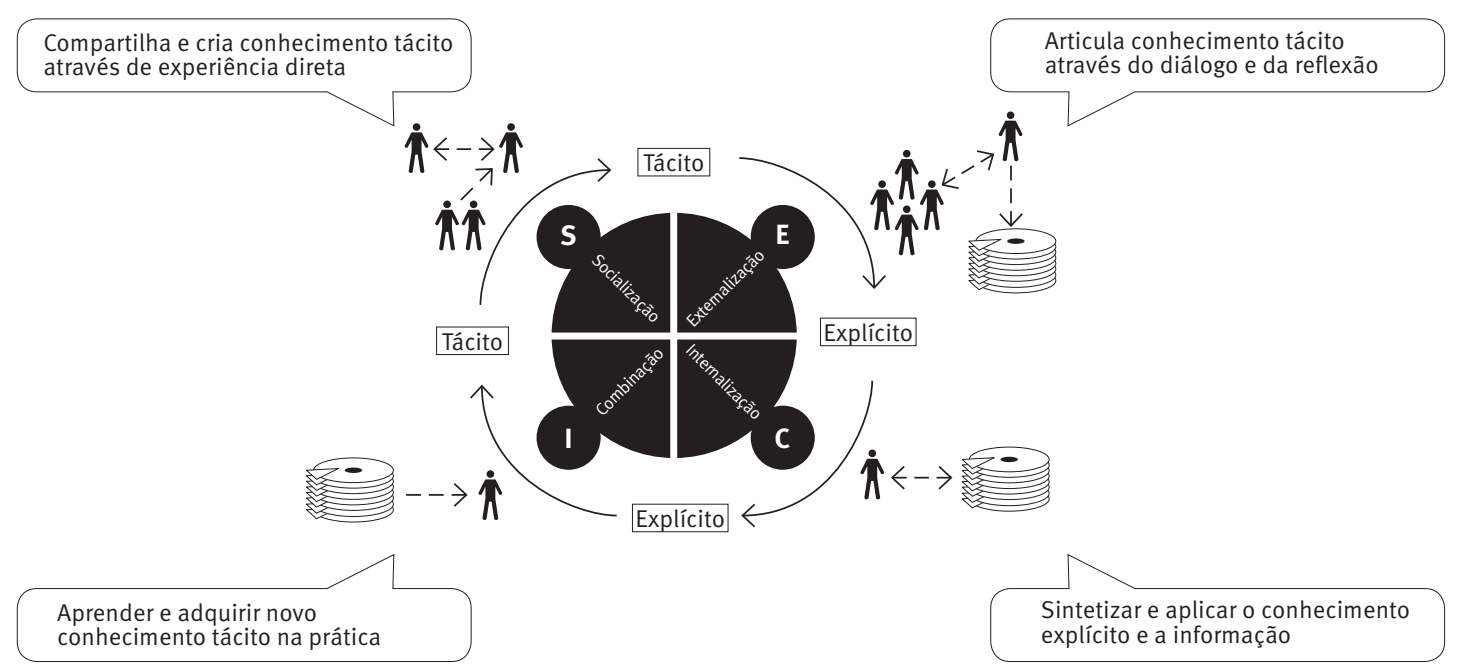

Fonte: Adaptado de Nonaka e Takeuchi (1997).

Para melhor entender o fenômeno da criação e compartiIhamento do conhecimento, é importante também atentar para o ambiente no qual a espiral se desenrola. Esse ambiente pode ser identificado como $\mathrm{Ba}$, conceito criado inicialmente pelo filósofo japonês Kitaro Nishida e adaptado por Nonaka e Takeuchi para o seu modelo de criação e transmissão do conhecimento (Nonaka et al, 2000; Nonaka \& Konno, 2005). O Ba é definido como um "espaço compartilhado que serve de fundação para a criação do conhecimento". O Ba é o espaço fenomenológico (físico e conceitual) formado pela coletividade envolvida no processo analisado e ligado a um $\mathrm{Ba}$ maior, formado pela coletividade de todas as coletividades menores, chamado Basho. De acordo com os autores, conhecimento desligado do seu $\mathrm{Ba}$, ou contexto, passa a ser mera peça de informação.

Hansen (1999) e Sorenson, Rivkin e Fleming (2006) elaboraram trabalhos de interesse para o presente estudo. Cada um, à sua maneira, investigou o compartilhamento de conhecimento novo para a empresa, sob a perspectiva das redes sociais, e todos concluíram que o grau de complexidade do conhecimento compartilhado tem papel importante no processo.

Hansen (1999) argumenta que o compartilhamento de conhecimento dá-se por via de "ligações fortes" (strong ties) ou de "ligações fracas" (weak ties). As primeiras (ligações fortes) são providas pelo aparato tecnológico da empresa e promove- 
riam, preferencialmente, a codificação e consolidação do conhecimento complexo (grande volume de dados, ou seja, "grande quantidade de ingredientes na receita”). As ligações fracas são asseguradas pelas interações na rede social e viabilizariam, em especial, o compartilhamento e captação do conhecimento simples (menor volume de dados, "receitas mais simples") entre pessoas e grupos de trabalho. Ligações fortes são normalmente conduzidas via infraestrutura tecnológica (sistemas de informação) e têm natureza explícita. Ligações fracas são procedidas nas relações interpessoais e têm, prioritariamente, caráter tácito. Para Hansen, os termos "forte" e "fraco" não têm relação com a importância da ligação, pois ligações fracas podem, eventualmente, ser mais importantes do que ligações fortes. Esses termos têm significados mais próximos, respectivamente, de "hard" e "soft". As conclusões de Hansen têm suporte em uma investigação empírica realizada em 120 projetos de desenvolvimento de novos produtos atribuídos a 41 grupos de trabatho de uma grande empresa transnacional do setor eletrônico. O parâmetro escolhido para aferir a efetividade do compartilhamento de conhecimento entre os grupos foi o tempo de desenvolvimento de um novo produto. Neste ponto, convém lembrar que a ideia de que as redes sociais são um instrumento efetivo para o compartilhamento de certos modos de conhecimento vem de Granovetter $(1973,1983)$, pesquisador que atribui uma enorme importância ao papel das interações entre pessoas na rede social, a ponto de subestimar a importância do aparato tecnológico no compartilhamento de conhecimento.

Sorenson et al. (2006) aproximam-se das conclusões de Hansen (1999), na medida em que reconhecem que a natureza do conhecimento compartilhado tem influência expressiva na efetividade daquele processo. Para os primeiros, conhecimentos simples seriam compartilhados com a mesma efetividade, quer venham de fontes próximas ou distantes. Por outro lado, conhecimentos complexos resistiriam ao compartilhamento, até mesmo no interior da rede social em que foram gerados.

\section{Análise de redes sociais}

A espiral do conhecimento origina-se e evolui a partir das relações de comunicação entre indivíduos. Para aferir de algum modo essas relações, a análise das redes sociais foi, assim, vista pelos autores deste trabalho como uma ferramenta com possibilidade de utilização no estudo de caso do BCAD. Para Wasserman e Faust (1999), uma rede social consiste em um conjunto finito de atores e as respectivas relações entre eles. "A presença de relação entre os atores é primordial para que um conjunto de atores seja definido como uma rede social" (Wasserman \& Faust, 1999, p. 20). Uma rede pode ser represen- tada por intermédio de uma estrutura gráfica, como um grafo, com nós interligados por linhas, em que os nós representam os atores e as linhas representam a relação entre eles. A Figura 2 exemplifica um grafo com seis nós (indivíduos), para os quais as linhas representam relacionamentos. Linhas cheias, tracejadas e pontilhadas representam diferentes tipos de relações, que podem ser simultaneamente identificadas em um mesmo grafo. Este é um exemplo de um grafo direto multivariado (Wasserman \& Faust, 1999).

Figura 2. Exemplo de grafo

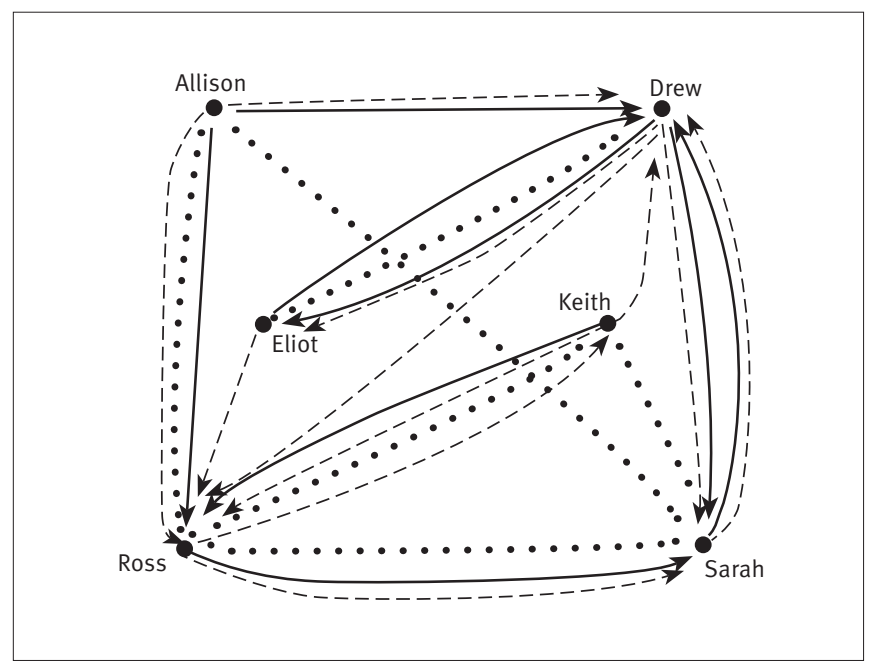

Fonte: Wasserman e Faust (1999).

No presente caso, há interesse nas relações em que ocorre compartilhamento de conhecimento, de acordo com as quatro conversões do modelo SECl. Aqui, a rede social focada é aquela criada pelos membros da equipe do BCAD, por ocasião da realização da etapa do DP do EcoSport sob sua responsabilidade. Como uma rede social não fica confinada exclusivamente a um departamento funcional, dela também fazem parte os indivíduos e grupos da organização que interagem com o BCAD em nível marginal.

Quando se analisa uma rede social, os fatores mais importantes a serem considerados são os atores e suas ações, as ligações e o modo como a informação flui entre eles. Esses são, igualmente, fatores de interesse do modelo $\mathrm{SECl}$, o que favorece a utilização do referido modelo em associação com a análise de redes sociais.

Para o desenho da rede social do BCAD, foi necessário identificar os atores da rede e os indivíduos com quem aqueles atores se relacionavam dentro das fronteiras funcionais da equipe, porém com certo grau de flexibilidade, de modo a permitir a inclusão de membros marginalmente ligados ao BCAD, mas pertencentes a outras equipes da planta da Ford em Camaçari. 
Cabe aqui recordar os significados dos termos, utilizados por Wasserman e Faust (1999), para a análise de redes sociais. Atores são as entidades que interagem na rede social. Podem ser indivíduos, grupos, subgrupos, unidades, corporações, organizações, coletividades, países ou outros agrupamentos sociais. 0 ator não é necessariamente um membro integrante da rede, porém deve atuar sobre a rede. Comumente adotam-se atores equalizados, ou seja, entidades do mesmo tipo, sejam indivíduos ou grupos, embora Galaskiewicz (1985) e Galaskiewicz e Wasserman (1989 apud como citado em Wasserman \& Faust, 1999) mencionem casos em que foram analisadas interações entre atores de categorias diferentes, como indivíduos versus grupos.

Laço é o conceito que descreve as relações entre os atores. Há inúmeros tipos de laços, porém o elemento mais importante de um laço é que este estabelece um elo qualquer entre dois atores. Alguns tipos de laço podem ser, por exemplo, amizade, carinho, respeito, associação, afiliação, interação comportamental, hierarquia e - o que é mais importante para esta pesquisa - a transferência e compartilhamento de recursos, sejam estes tangíveis ou intangíveis. No caso do compartilhamento de conhecimento, as redes estão transferindo um recurso de natureza intangível.

Grupo é um sistema finito de atores que se inter-relacionam socialmente. Um grupo pode ser definido com base em relações hierárquicas (caso do BCAD) ou não. Um grupo pode interagir com indivíduos e grupos externos. Delimitar com exatidão o grupo é uma necessidade da análise de redes sociais. Enquanto grupo é o conjunto dos atores de uma rede social, relação é a trama composta pelos laços existentes entre os atores. No caso estudado, o compartilhamento de experiências e conhecimento entre os atores do grupo forma a relação entre eles. Do mesmo modo como é necessário delimitar grupos, quando se trata de relações, é preciso igualmente delimitar a natureza e amplitude das relações analisadas, pois dois atores podem, eventualmente, ter mais relações do que aquelas captadas pela análise. Isso se deve ao fato de que muitas das relações não podem ser analisadas (ou não se tem interesse em fazê-lo) utilizando-se as ferramentas escolhidas, na ocasião, para capturar os dados. No estudo aqui apresentado, focaram-se as relações criadas especificamente pelo compartilhamento de conhecimento voltado ao trabalho executado no dia a dia da equipe do $B C A D$, em detrimento de outras relações sociais, de amizade ou mesmo familiares.

Centralidade é a qualidade de um ator que se relaciona com vários atores dentro da rede, desconsiderando-se a direção dessas relações, ou seja, quanto mais relações, de quaisquer tipos, tiver um ator com outros atores, maior a sua centra- lidade. No caso estudado, um ator pode ser central recebendo conhecimento de vários atores e/ou transmitindo conhecimento para vários atores. Esse nível de relação torna-o mais visível diante dos demais atores. Centralidade, portanto, não denota necessariamente um julgamento de valor utilitário. Em outras palavras, o ator mais central, no caso desta investigação, não seria necessariamente aquele que protagoniza mais conversões e transmite mais conhecimento. 0 ator que possui mais relações na rede é normalmente representado no centro do grafo, que se estenderá para as extremidades, de modo gradativamente decrescente em termos de quantidade de laços para cada nó.

Prestígio é a qualidade de um ator que se relaciona com vários outros atores, porém, ao contrário da centralidade, para o prestígio, a direção dos laços é importante. Para o estudo de caso aqui descrito, prestígio poderá ser uma métrica para identificar aqueles que mais transmitem conhecimento à equipe, os quais despontarão como referências reconhecidas mais frequentemente pelos demais atores; assim, quanto mais relações de compartilhamento de conhecimento o ator possuir, maior o seu prestígio na rede.

Na seção que se segue, serão expostos os critérios metodológicos empregados para avaliar as questões da natureza e do impacto do compartilhamento de conhecimento durante o estágio do projeto de desenvolvimento do novo EcoSport sob responsabilidade do BCAD.

\section{CRITÉRIOS METODOLÓGICOS}

A fim de dar resposta às questões relativas ao tipo e efetividade do compartilhamento de conhecimento entre os participantes do projeto investigado, os seguintes passos foram adotados:

- Mapear os relacionamentos formais mantidos na equipe (rede formal);

- Mapear a rede social informal da equipe;

- Registrar os tipos de compartilhamento de conhecimentos preferidos pelos membros da equipe, com base nos modos de conversão (socialização, externalização, combinação, internalização); e

- Registrar a efetividade do compartilhamento de conhecimento, na percepção dos integrantes da equipe.

Para o mapeamento dos relacionamentos formais ocorridos no BCAD (primeiro passo da metodologia utilizada nesta investigação), foi empregada a documentação que descreve a organização interna da planta automotiva da Ford em Camaçari. Para a realização dos três passos mencionados, empregou-se, como instrumento, um questionário estruturado aplicado aos 
indivíduos que participaram do desenvolvimento do EcoSport no BCAD, em que as informações a seguir foram obtidas, com base na percepção dos respondentes:

a. Relações entre nós, expressas como atividades funcionais da equipe;

b. Atores que mais compartilharam conhecimento na equipe;

c. Indicações dos nós externos de outras equipes da companhia que interagem com o BCAD;

d. Atores considerados membros disseminadores de conhecimentos;

e. Tipos preferidos de compartilhamento de conhecimentos (escolha feita por ordem de preferência, entre 20 alternativas oferecidas pelo questionário);

f. Frequência relativa de cada um dos quatro tipos de conversão de conhecimentos praticados, aferida com escala de gradação inversa do tipo Likert, variando de 1 (alta frequência) a 5 (baixa frequência); e

g. Efetividade dos tipos de conversão de conhecimentos, utilizando-se, para aferição, uma escala do tipo Likert, semelhante à anterior, variando de 1 (alta efetividade) a 5 (baixa efetividade).

0 mapeamento da rede social informal do BCAD foi realizado com o uso do software Ucinet $\bigodot$, cujo emprego mostrouse satisfatório em análises semelhantes à do presente estudo (Borgatti, Everett, \& Freeman, 2002). O software Ucinet integrado com o Netdraw é uma ferramenta que tem a vantagem de possuir funcionalidades de gerar uma visualização gráfica da rede (descrição no site https://sites.google.com/site/ucinetsoftware/home, acessado em julho de 2012).

\section{RESULTADOS}

O organograma da Figura 3 ajuda a entender os relacionamentos formais mantidos na equipe de projeto. Ele ilustra a divisão de tarefas durante a maior parte do desenvolvimento do novo EcoSport, utilizando-se a codificação N(n) própria da Ford para identificar os postos de trabalho dos integrantes da equipe do BCAD. Na estrutura organizacional, a posição N2o corresponde ao supervisor da equipe. N3 executou tarefas de revisão, trabalhando nos modelos CAD de todo o carro. N24, N14, N1, N13, N12, N10 e N6 cumpriram suas tarefas como líderes de setor, executando também algumas tarefas de modelagem CAD. Os demais responderam, respectivamente, aos líderes supracitados, executando as tarefas de detalhamento da modelagem CAD, conforme a estrutura da Figura 3. Optou-se por não nomear, aqui, os setores de atividade, a fim de que a identidade dos integrantes da equi- pe seja preservada. Adotou-se, também, a codificação $M(n)$ para nomear os integrantes da companhia não pertencentes ao BCAD, porém com atuação sobre aquela equipe, conforme se verá adiante. Esses atores externos somente aparecerão na rede informal, uma vez que não faziam parte da hierarquia formal do $B C A D$, por pertencerem a outras equipes da companhia.

Figura 3. Organograma do BCAD - A rede formal do BCAD

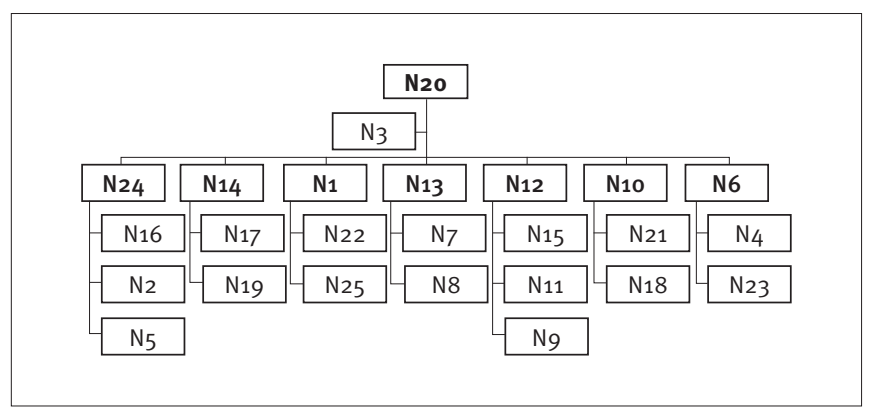

Nota: Baseado na estrutura organizacional da Ford, Camaçari.

A equipe iniciou sua atuação no projeto em 2009, com 15 integrantes, e concluiu sua participação em 2011, com 21 participantes formais. Com essa estrutura organizacional, o BCAD assumiu a incumbência de realizar as tarefas descritas brevemente a seguir.

A equipe foi responsável pelo desenvolvimento de cerca de 200 peças em chapa de aço (sheetmetal) para a carroceria do novo veículo durante o período do projeto. Essa atividade compreendeu o desenho de portas laterais, capô, tampa do porta-malas, bem como de toda a estrutura lateral do veículo e do seu teto. Para tanto, trabalhou-se com um software de modelagem tridimensional (3D) chamado CATIA, da empresa francesa Dassault, e com o software TEAMCENTER, da empresa alemã Siemens. Esse último era um aplicativo do tipo Product Lifecycle Management (PLM), essencialmente um gerenciador para os arquivos $C A D$, com a funcionalidade, entre outras, de controle de versões de arquivos.

O desenvolvimento deu-se em três macroetapas, para cada uma das quais o BCAD tinha que entregar todas as peças modeladas em 3D, com níveis de maturidade crescentes, até atingir-se o milestone da terceira e última etapa, em que todos os modelos alcançariam sua forma final. Além do modelamento das peças em sheetmetal, o BCAD foi responsável pelo gerenciamento e desenvolvimento das chamadas master sections do carro, em desenhos bidimensionais (2D), que representam as principais interfaces entre as peças da carroceria e as demais peças do carro, com o objetivo de preservar interfaces com descontinuidades mínimas e subsidiar o Estú- 
dio de Design no desenvolvimento das exigências de estilo do novo veículo. As master sections começaram a ser desenvolvidas na primeira das três macroetapas, paralelamente à modelagem 3D das peças, e seguiram sendo desenvolvidas ao longo de todo o projeto.

A Figura 4 mostra as principais equipes que faziam interfaces com o BCAD. Para as denominações das equipes, mantiveram-se os termos correntemente usados na companhia, pois pareceu aos autores deste trabalho que uma tradução dos referidos termos para o vernáculo poderia enfraquecer a descrição. Destacam-se o Estúdio de Design, responsável, como foi dito, pelo estilo do veículo e de suas qualidades estéticas e simbólicas, e as Project Management Teams (PMTs). As PMTs são constituídas pelas equipes de Body Closures e Body Structures, responsáveis pelas peças da carroceria do veículo, bem como pelas equipes de Exterior Trim e Interior Trim, responsáveis, respectivamente, pelas peças plásticas do exterior e do interior do veículo, tais como para-choques, grades, molduras, painel, console central, bancos e outras. Outras equipes funcionais com interfaces relevantes com o BCAD são a CAD do Chassi (CCAD), responsável pelo CAD das peças do underbody (parte inferior da carroceria); e o grupo de Basic Design, composto pelas equipes de Occupant Package e Mechanical Package, responsáveis pelas especificações ergonômicas do carro. Além desses grupos de trabalho, o BCAD tem interface com a equipe de Gerenciamento de Programas, responsável pelo gerenciamento -top level do projeto do novo EcoSport.

Figura 4. Interfaces do Body CAD no DP da Ford - Camaçari

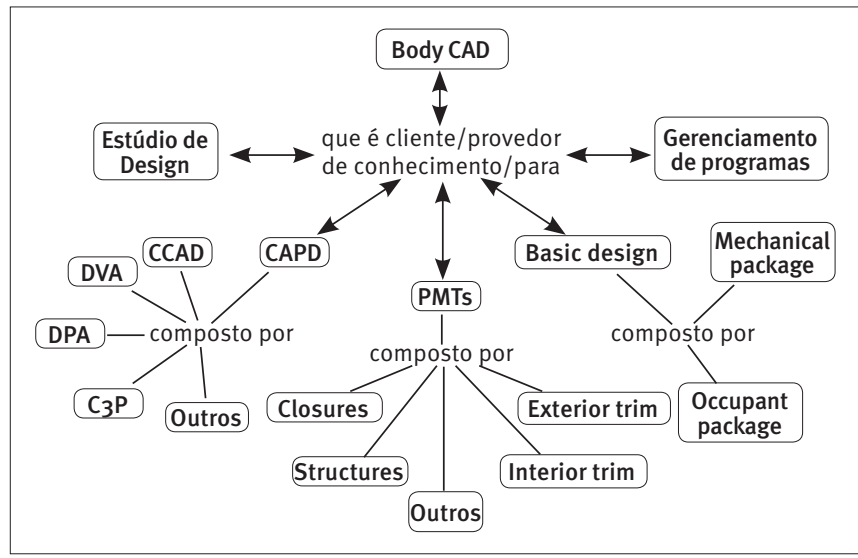

Nota: Baseada na estrutura organizacional da Ford, Camaçari.

\section{A rede social informal do BCAD}

Para a coleta de dados destinados à elaboração da rede social informal da equipe do BCAD, foi solicitado, a cada um dos 21 participantes da equipe (todos foram consultados), que indicasse no questionário os indivíduos procurados por eles para a obtenção de conhecimentos sobre assuntos específicos do cotidiano do trabalho da equipe. Os principais temas e disciplinas técnicos foram listados no questionário, a fim de facilitar as respostas. Com esses dados, foi possível mapear a rede informal constituída pelos atores internos ( $\mathrm{N}_{1}$ a N21, vistos na Figura 5).

Considerando-se os valores binários para os laços entre os atores, percebe-se, na rede, que os atores dos nós N14, N6 e N12 são aqueles de maior prestígio (ver Tabela 1, para os índices de prestígio). Os índices de prestígio aqui apresentados foram calculados automaticamente pelo software Ucinet, com base na estrutura da rede. Os três referidos atores de maior prestígio eram líderes de setor durante o projeto do novo EcoSport, confirmando-se, portanto, a identidade de posição de prestígio daqueles indivíduos nas redes formal e informal.

\section{Figura 5. Rede social informal do BCAD - Atores internos}

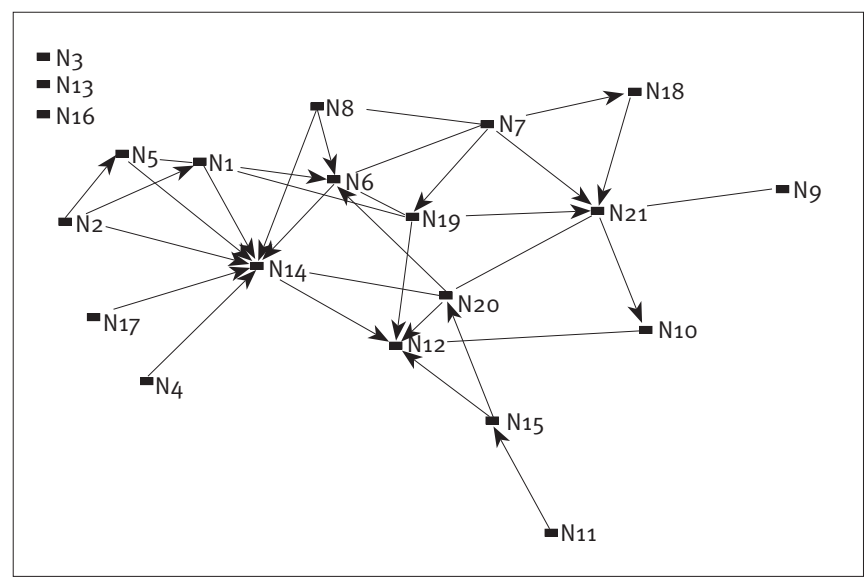

Nota: Baseada no software Ucinet.

Percebem-se, também, na rede da Figura 5, três atores (N3, N13 e N16) que não mencionaram integrante algum da equipe como suas fontes de conhecimento durante o projeto, estando, portanto, fora da rede. Estes mesmos atores passam a integrar a rede, entretanto, quando são incluídos nela os atores externos à equipe. Esses últimos atores, identificados pela codificação $M(n)$, são reconhecidos como fontes de conhecimento dos primeiros (Figura 6). A mencionada figura representa 0 conjunto completo de atores (internos e externos) envolvidos no compartilhamento de conhecimento do estágio do DP sob a responsabilidade do BCAD. Observando-se, mais uma vez, a rede social informal completa do BCAD (Figura 6) e consultando-se agora a Tabela 2, alguns fatos interessantes destacam-se e são comentados a seguir. 
TABELA 1. Atores internos da rede social informal, ordenados por prestígio

\begin{tabular}{|c|c|}
\hline Ator (Integrantes de BCAD) & Índice de prestígio \\
\hline $\mathrm{N} 14$ & 10 \\
\hline N6 & 5 \\
\hline $\mathrm{N} 12$ & 5 \\
\hline $\mathrm{N} 21$ & 4 \\
\hline $\mathrm{N} 20$ & 2 \\
\hline $\mathrm{N}_{1}$ & 2 \\
\hline N19 & 1 \\
\hline $\mathrm{N}_{5}$ & 1 \\
\hline N8 & 1 \\
\hline $\mathrm{N}_{15}$ & 1 \\
\hline $\mathrm{N} 18$ & 1 \\
\hline $\mathrm{N} 10$ & 1 \\
\hline $\mathrm{N}_{7}$ & 0 \\
\hline $\mathrm{N} 20$ & 0 \\
\hline $\mathrm{N}_{4}$ & 0 \\
\hline N17 & 0 \\
\hline N11 & 0 \\
\hline Ng & 0 \\
\hline $\mathrm{N}_{3}$ & 0 \\
\hline $\mathrm{N} 13$ & 0 \\
\hline $\mathrm{N} 16$ & 0 \\
\hline
\end{tabular}

Nota: Baseada no software Ucinet.

Figura 6. Rede social informal do BCAD - atores internos e externos

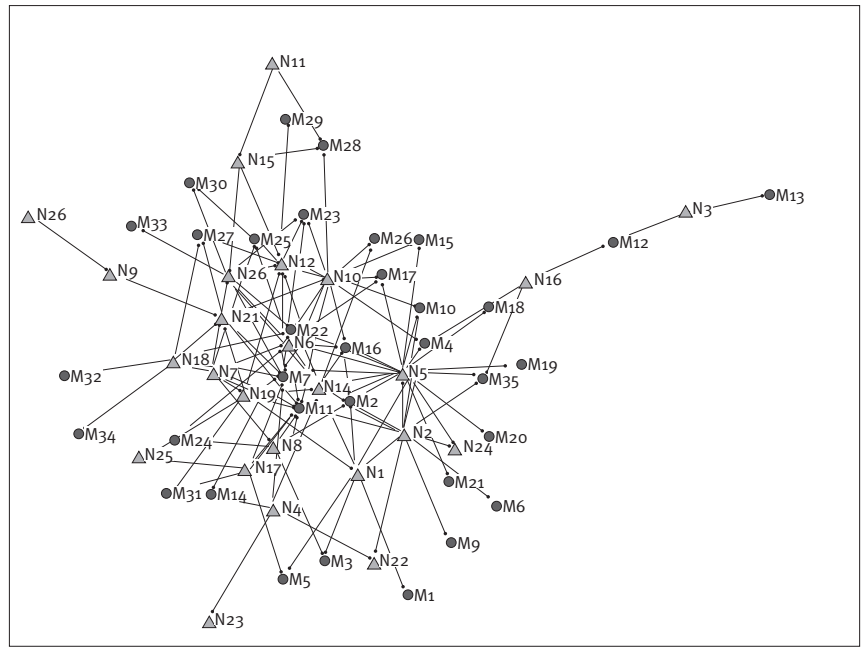

Nota: Baseada no software Ucinet.
M7 é o ator de maior prestígio dentro da rede, embora não seja integrante da equipe do BCAD. Ele exerce uma atividade conhecida na companhia como Tech Spec, ou seja, é um especialista técnico, cuja missão é exatamente disseminar conhecimento sobre aspectos técnicos relacionados ao trabalho de desenvolvimento do produto Ford, provendo meios para a solução de problemas técnicos. Para facilitar a visualização, na Figura 6, os atores externos, $M(n)$, estão representados pelos círculos em cinza-escuro, enquanto os triângulos em cinza-claro representam os atores internos do BCAD.

\section{TABELA 2. Atores da rede social informal completa por ordem decrescente de prestígio}

\begin{tabular}{|c|c|}
\hline $\begin{array}{c}\text { Atores } \\
\text { (internos e externos) }\end{array}$ & Índice de prestígio \\
\hline M7 & 12 \\
\hline M11 & 11 \\
\hline $\mathrm{N} 14$ & 10 \\
\hline$M 22$ & 6 \\
\hline N6 & 5 \\
\hline $\mathrm{N} 12$ & 5 \\
\hline $\mathrm{M} 2$ & 5 \\
\hline M4 & 5 \\
\hline $\mathrm{N} 21$ & 4 \\
\hline M16 & 4 \\
\hline M23 & 4 \\
\hline M25 & 4 \\
\hline M10 & 3 \\
\hline M17 & 3 \\
\hline$M 27$ & 3 \\
\hline $\mathrm{M} 28$ & 3 \\
\hline M35 & 3 \\
\hline $\mathrm{N}_{1}$ & 2 \\
\hline $\mathrm{N} 20$ & 2 \\
\hline $\mathrm{N} 22$ & 2 \\
\hline
\end{tabular}

Nota: Baseada no software Ucinet. 


\section{Vinte primeiros resultados}

De modo geral, entre as menções feitas pelos entrevistados, percebe-se uma importante participação dos integrantes de outras equipes de projeto, em especial DVA e $\mathrm{C}_{3} \mathrm{P}$, por intermédio dos nós M11, M22, M2, M16, M25, M10 e M17 (Tabela 2), evidenciando que esses sete atores externos estão entre os 20 atores da rede completa com maior prestígio.

Percebe-se, porém, a ausência de integrantes do Estúdio de Design, do Basic Design e do Gerenciamento de Programas na rede completa, o que indica uma relação fraca entre essas equipes e a equipe do BCAD, fato preocupante em razão da importância dos relacionamentos com as referidas equipes para o sucesso do trabalho do BCAD.

Como informação acessória, indicam-se, na Tabela 3, os índices globais da rede social informal completa do BCAD, obtidos com o software Ucinet ${ }^{\odot}$.

\section{TABELA 3. Índices globais da rede social informal completa}

\begin{tabular}{l|l}
\hline Índice & Valor \\
\hline Número de componentes & 59 \\
\hline Centralidade média & 2,300 \\
\hline Densidade & 0,039 \\
\hline
\end{tabular}

Nota: Baseada no Software Ucinet.

\section{Tipos de compartilhamento de conhecimentos preferidos}

Para aferir os tipos de compartilhamento de conhecimentos preferidos pelos membros do BCAD, utilizaram-se, como referência, os modos de conversão do modelo SECl.

Foram apresentadas no questionário 20 situações de compartilhamento de conhecimento do cotidiano da equipe, entre as quais os indivíduos tiveram que selecionar suas preferidas. Essas 20 situações estavam divididas equitativamente entre os quatro modos de conversão, cabendo, assim, para cada modo, cinco situações. Os respondentes não tinham, entretanto, conhecimento de que aquelas situações estavam categorizadas de acordo com os modos de conversão do modelo SECI. Para eles, tratava-se, simplesmente, de situações do cotidiano funcional da equipe.

Achou-se conveniente, ainda, separar as 20 referidas situações em dois grupos, cada um com 10 situações, e apresentá-las, assim separadas, aos respondentes. 0 primeiro grupo, correspondente aos modos de socialização e internalização, foi denominado, pelos pesquisadores, "métodos de captação de conhecimento", enquanto o segundo grupo, formado pelos modos de externalização e combinação, foi denominado "métodos de transmissão de conhecimento". A intenção foi a de distinguir, respectivamente, os fluxos de captação e de transmissão de conhecimentos. Do mesmo modo, os respondentes não tinham conhecimento dessa intenção dos pesquisadores. Os respondentes foram solicitados apenas a numerar as situações de cada grupo, de 1 a 10, sendo 1 o tipo de compartilhamento de conhecimento mais fortemente preferido e 100 menos preferido.

Enquanto alguns respondentes assinalaram todas as 10 situações de cada grupo, outros assinalaram quantidades menores de situações, uma evidência de que as percepções dos indivíduos variavam em conteúdo e intensidade. Para uniformizar o tratamento dos dados e ampliar o aproveitamento de respostas, foram consideradas as preferências até o limite de cinco indicações por respondente.

\section{Métodos de captação de conhecimentos (socialização e internalização)}

Para os métodos de captação de conhecimentos (Tabela 4), foram computadas 100 indicações de preferências válidas, sendo $56 \%$ delas $(56 / 100)$ por modos de socialização para a captação de conhecimento e $44 \%$ (44/100) por modos de internalização para a captação de conhecimento durante o projeto do novo EcoSport. Entradas marcadas com (-) indicam respostas omitidas ou anuladas.

Pela Tabela 4, observa-se, também, que, dos 20 participantes da enquete com respostas válidas, 16 consideraram um modo de socialização como sua primeira escolha para receber conhecimento. Um integrante $\left(\mathrm{N}_{1} \mathrm{O}\right)$ enumerou, simultaneamente, um modo de socialização e um modo de internalização como primeira escolha, manifestando igual preferência por ambos.

\section{Métodos de transmissão de conhecimentos (externalização e combinação)}

Para os métodos de transmissão de conhecimentos (Tabela 5), foram consideradas 74 respostas válidas, refletindo uma preferência de 63,5\% (47/74) por modos de externalização e 36,5\% (27/74) por modos de combinação. Das 18 respostas válidas, 15 apontaram um modo de externalização como primeira escolha para transmitir conhecimento durante o projeto do novo EcoSport. Entradas marcadas com (-) indicam respostas omitidas ou anuladas. Um participante $\left(\mathrm{N}_{17}\right)$ enumerou um modo de socialização e um modo de internalização como simultaneamente preferidos, denotando preferência idêntica por ambos. 
TABELA 4. Preferência por métodos de captação de conhecimentos

\begin{tabular}{|c|c|c|c|}
\hline Ator & $P(s)$ & $\mathrm{P}(\mathrm{i})$ & $\begin{array}{c}1^{0} \text {. Modo } \\
\text { selecionado }\end{array}$ \\
\hline $\mathrm{N}_{1}$ & 1 & 4 & I \\
\hline $\mathrm{N} 2$ & 4 & 3 & $S$ \\
\hline $\mathrm{N}_{3}$ & 2 & 3 & I \\
\hline $\mathrm{N}_{4}$ & 3 & 2 & $S$ \\
\hline $\mathrm{N}_{5}$ & 3 & 2 & $S$ \\
\hline N6 & 2 & 3 & $S$ \\
\hline $\mathrm{N}_{7}$ & 4 & 3 & $S$ \\
\hline N8 & 4 & 1 & $S$ \\
\hline N9 & 3 & 1 & $S$ \\
\hline $\mathrm{N}_{10}$ & 2 & 3 & $S / I$ \\
\hline $\mathrm{N}_{11}$ & 2 & 1 & $S$ \\
\hline $\mathrm{N}_{12}$ & 3 & 2 & $S$ \\
\hline $\mathrm{N}_{13}$ & - & - & - \\
\hline $\mathrm{N}_{14}$ & 2 & 3 & I \\
\hline N15 & 1 & 2 & I \\
\hline $\mathrm{N}_{16}$ & 3 & 2 & $S$ \\
\hline N17 & 2 & 2 & $S$ \\
\hline $\mathrm{N} 18$ & 3 & 2 & $S$ \\
\hline N19 & 4 & 1 & $S$ \\
\hline $\mathrm{N} 2 \mathrm{O}$ & 4 & 1 & $S$ \\
\hline \multirow[t]{2}{*}{$\mathrm{N}_{21}$} & 4 & 3 & $S$ \\
\hline & 56 & 44 & $S=16 / I=5$ \\
\hline
\end{tabular}

P (s) - Preferência por socialização / P (i) - Preferência internalização

Em resumo, tem-se que, durante o projeto do novo EcoSport (Tabela 6), os membros da equipe BCAD preferiram utilizar modos de socialização em $56 \%$ das situações para receber conhecimentos e preferiram utilizar modos de externalização em $63,5 \%$ das situações para transmitir conhecimentos. Além disso, é notória a opção por um modo de socialização como primeira escolha para recebimento de conhecimento e de um modo de externalização como primeira escolha para transmissão de conhecimento.
TABELA 5. Preferência por métodos de transmissão de conhecimentos

\begin{tabular}{|c|c|c|c|}
\hline Ator & $P(e)$ & $P(c)$ & $\begin{array}{c}1^{0} \stackrel{0}{ } \text {. Modo } \\
\text { selecionado }\end{array}$ \\
\hline $\mathrm{N}_{1}$ & 1 & 3 & C \\
\hline $\mathrm{N} 2$ & - & - & - \\
\hline $\mathrm{N}_{3}$ & 3 & 2 & $E$ \\
\hline $\mathrm{N}_{4}$ & 3 & 1 & $E$ \\
\hline $\mathrm{N}_{5}$ & 3 & 2 & $E$ \\
\hline N6 & 2 & 3 & E \\
\hline N7 & 4 & 2 & E \\
\hline N8 & 4 & 1 & $E$ \\
\hline N9 & 2 & 1 & E \\
\hline $\mathrm{N}_{10}$ & 3 & 1 & $E$ \\
\hline$N_{11}$ & - & - & - \\
\hline $\mathrm{N}_{12}$ & 3 & 2 & $E$ \\
\hline$N_{13}$ & 3 & 2 & $\mathrm{E}$ \\
\hline $\mathrm{N}_{14}$ & 3 & 2 & $\mathrm{E}$ \\
\hline $\mathrm{N}_{15}$ & - & - & - \\
\hline N16 & 3 & 0 & $E$ \\
\hline $\mathrm{N} 17$ & - & - & $C / E$ \\
\hline $\mathrm{N} 18$ & 2 & 1 & C \\
\hline N19 & 3 & 1 & $E$ \\
\hline $\mathrm{N} 2 \mathrm{O}$ & 2 & 3 & C \\
\hline \multirow[t]{2}{*}{$\mathrm{N} 21$} & 3 & 0 & $E$ \\
\hline & 47 & 27 & $E=15 / C=4$ \\
\hline
\end{tabular}

$P(e)$ - Preferência por externalização / P (c) - Preferência por combinação

\section{TABELA 6. Percentual de preferência por modo de} conversão

\begin{tabular}{l|l|l|l|l}
\hline & \multicolumn{2}{|l|}{ Captação de conhecimento } & \multicolumn{2}{l}{\begin{tabular}{l}
\multicolumn{2}{l}{ Transmissão de } \\
conhecimento
\end{tabular}} \\
\hline & $\mathrm{P}(\mathrm{s})$ & $\mathrm{P}(\mathrm{i})$ & $\mathrm{P}(\mathrm{e})$ & $\mathrm{P}(\mathrm{c})$ \\
\hline BCAD & $56 \%$ & $44 \%$ & $63,50 \%$ & $36,50 \%$ \\
\hline
\end{tabular}


TABELA 7. Frequência e Percepção de Efetividade dos métodos de captação de conhecimentos

\begin{tabular}{|c|c|c|c|c|}
\hline Ator & $\mathrm{F}(\mathrm{s})$ & $\mathrm{F}(\mathrm{i})$ & $E(s)$ & $E(i)$ \\
\hline $\mathrm{N}_{1}$ & 1,00 & 1,50 & 1,00 & 2,50 \\
\hline $\mathrm{N} 2$ & 1,00 & 2,00 & 1,25 & 2,60 \\
\hline $\mathrm{N}_{3}$ & 2,00 & 2,33 & 2,00 & 3,00 \\
\hline$N_{4}$ & 1,33 & 3,50 & 2,33 & 3,00 \\
\hline $\mathrm{N}_{5}$ & 1,66 & 2,00 & 2,00 & 3,00 \\
\hline N6 & 3,50 & 3,33 & 2,50 & 2,00 \\
\hline N7 & 2,00 & 3,33 & 1,75 & 2,66 \\
\hline N8 & 2,75 & 3,00 & 2,33 & 3,00 \\
\hline N9 & 2,00 & 2,00 & 2,33 & 3,00 \\
\hline $\mathrm{N}_{10}$ & 1,50 & 2,66 & 1,50 & 1,66 \\
\hline $\mathrm{N}_{11}$ & 1,00 & 3,00 & 1,50 & 3,00 \\
\hline $\mathrm{N}_{12}$ & 1,33 & 2,00 & 1,33 & 1,00 \\
\hline $\mathrm{N}_{13}$ & - & - & - & - \\
\hline $\mathrm{N}_{14}$ & 2,00 & 2,00 & 4,00 & 3,50 \\
\hline $\mathrm{N}_{15}$ & 3,00 & 2,50 & 3,00 & 1,00 \\
\hline $\mathrm{N}_{16}$ & 1,00 & 1,00 & 2,33 & 1,50 \\
\hline $\mathrm{N}_{17}$ & 2,00 & 1,00 & 1,00 & 1,00 \\
\hline $\mathrm{N}_{18}$ & 1,33 & 3,50 & 2,00 & 2,50 \\
\hline $\mathrm{N}_{19}$ & 2,50 & 2,00 & 2,00 & 2,00 \\
\hline $\mathrm{N} 2 \mathrm{O}$ & 2,75 & 3,00 & 1,75 & 2,00 \\
\hline $\mathrm{N} 21$ & 1,25 & 2,00 & 2,00 & 2,00 \\
\hline Médias & 1,85 & 2,38 & 2,00 & 2,30 \\
\hline
\end{tabular}

F (s) - Frequência de Socialização

F (i) - Frequência de Internalização

E (s) - Percepção de efetividade dos modos de Socialização

E (i ) - Percepção de efetividade dos modos de Internalização

\section{Frequência e efetividade do compartilhamento de conhecimentos}

Os respondentes do questionário foram solicitados, também, a identificar, em uma escala proporcional de 1 a 5 (sendo 1 "diariamente" e 5 "nunca"), com que frequência utilizaram, respectivamente, os métodos de captação e transmissão de conhecimentos já identificados por eles próprios como preferenciais. Ao mesmo tempo, os referidos métodos foram também carac- terizados pelos respondentes quanto à efetividade, em uma escala semelhante, em que 1 indicava "sucesso absoluto" e 5 , "insucesso total". Desse modo - e tendo-se em conta as limitações da aferição - supõe-se terem sido aferidas, em uma escala de gradação proporcional, as percepções dos respondentes quanto à efetividade do compartilhamento de conhecimentos durante o projeto do novo EcoSport. Recorde-se que as escalas aqui utilizadas têm intensidades decrescentes, de modo que valores numéricos mais baixos, tanto para frequência quanto para efetividade, sinalizam melhores resultados.

A Tabela 7 mostra os resultados dessa aferição para os métodos de captação de conhecimentos (entradas marcadas com (-) indicam respostas omitidas ou anuladas). Observe-se que os resultados apontam para uma frequência média elevada de utilização dos modos de socialização $(1,85)$, quando comparada com a frequência média dos modos de internalização $(2,38)$. A Tabela 7 destaca também que, em média, a efetividade percebida para os modos de socialização $(2,00)$ foi mais expressiva do que para os modos de internalização $(2,30)$. Esses resultados são congruentes com os do levantamento de preferências entre os referidos modos, que apontaram maior preferência por modos baseados em socialização do que por modos baseados em internalização para a obtenção de conhecimentos.

De modo semelhante, a Tabela 8 mostra os resultados para os métodos de transmissão de conhecimentos (entradas marcadas com (-) indicam respostas omitidas ou anuladas). Os dados mostram uma frequência média mais alta de utilização dos modos de externalização $(2,07)$, em comparação com os modos de combinação $(3,15)$. A percepção de efetividade dos modos baseados em externalização $(1,79)$ foi mais expressiva do que a dos modos baseados em combinação $(2,74)$. Esses resultados são igualmente congruentes com a preferência entre os métodos, que apontou os modos de externalização como preferidos, em comparação com os modos de combinação para a transmissão de conhecimentos.

Por fim, a Tabela 9 mostra, em ordem decrescente de intensidade, o ranking da efetividade média percebida pelos profissionais do BCAD consultados, para cada uma da $20 \mathrm{si}-$ tuações de compartilhamento de conhecimentos a eles apresentada por ocasião da consulta realizada no escopo deste trabalho. Confirma-se que as situações mais intensivas, respectivamente, nos modos de socialização (S) e externalização (E), foram percebidas como mais efetivas no compartilhamento de conhecimentos. De fato, entre as cinco primeiras situações do ranking de efetividade (Tabela 9), percebe-se que três delas referem-se a modos de socialização (S) e duas, a modos de externalização (E). 
TABELA 8. Frequência e Percepção de Efetividade dos métodos de transmissão de conhecimentos

\begin{tabular}{|c|c|c|c|c|}
\hline Ator & $\mathrm{F}(\mathrm{e})$ & $\mathrm{F}(\mathrm{c})$ & $E(e)$ & $E(c)$ \\
\hline $\mathrm{N}_{1}$ & 3,00 & 3,00 & 2,00 & 2,33 \\
\hline $\mathrm{N} 2$ & - & - & - & - \\
\hline $\mathrm{N}_{3}$ & 2,66 & 3,00 & 4,00 & 4,50 \\
\hline $\mathrm{N}_{4}$ & 1,66 & 4,00 & 1,33 & 3,00 \\
\hline $\mathrm{N}_{5}$ & 3,00 & 3,50 & 3,66 & 2,50 \\
\hline N6 & 1,00 & 3,66 & 1,50 & 1,66 \\
\hline $\mathrm{N}_{7}$ & 1,50 & 2,00 & 1,25 & 1,50 \\
\hline N8 & 1,25 & 3,00 & 1,25 & 3,00 \\
\hline N9 & 3,00 & 3,00 & 2,00 & 3,00 \\
\hline $\mathrm{N}_{10}$ & 1,33 & 3,00 & 1,00 & 3,00 \\
\hline $\mathrm{N}_{11}$ & - & - & - & - \\
\hline $\mathrm{N}_{12}$ & 1,00 & 2,00 & 1,00 & 1,00 \\
\hline $\mathrm{N}_{13}$ & - & - & - & - \\
\hline $\mathrm{N}_{14}$ & 2,66 & 2,00 & 2,00 & 2,50 \\
\hline $\mathrm{N}_{15}$ & 2,33 & 3,00 & 1,00 & 2,00 \\
\hline $\mathrm{N} 16$ & 1,00 & 5,00 & 1,00 & 5,00 \\
\hline N17 & - & - & - & - \\
\hline $\mathrm{N}_{18}$ & 2,00 & 2,00 & 2,00 & 2,00 \\
\hline N19 & 2,00 & 3,00 & 2,00 & 2,00 \\
\hline $\mathrm{N} 2 \mathrm{O}$ & 2,50 & 3,33 & 1,50 & 2,66 \\
\hline $\mathrm{N} 21$ & 3,33 & 5,00 & 2,00 & 5,00 \\
\hline Médias & 2,07 & 3,15 & 1,79 & 2,74 \\
\hline
\end{tabular}

F (e) - Frequência de Externalização

E (e) - Percepção de efetividade dos modos de Externalização

$F(c)$ - Frequência de Combinação

E (c) - Percepção de efetividade dos modos de Combinação

\section{CONSIDERAÇÕES FINAIS}

Este texto encerra-se mencionando alguns ensinamentos deixados pela aferição da experiência de compartilhamento de conhecimentos entre os membros de uma das equipes de desenvolvimento do novo EcoSport na Ford do Brasil, o BCAD.

Em primeiro lugar, percebe-se que a rede social interna do BCAD, embora satisfatoriamente responsiva na etapa do projeto do novo EcoSport sob sua responsabilidade, pode ser melhorada. Há membros que ainda se consideram isolados do processo de compartilhamento de conhecimentos, com poucos
TABELA 9. Ranking da efetividade das situações de compartilhamento de conhecimento segundo os integrantes do BCAD

\begin{tabular}{|c|c|}
\hline Situações de compartilhamento de conhecimentos & $\begin{array}{l}\text { Efetividade } \\
\text { média }\end{array}$ \\
\hline Criar arquivo CAD como referência (E) & 1,73 \\
\hline Consultar colegas mais experientes (S) & 1,80 \\
\hline Consultar engenheiros das peças (S) & 1,84 \\
\hline Compartilhar via e-mail desdobramentos do trabalho (S) & 2,00 \\
\hline $\begin{array}{l}\text { Enviar imagens apresentando problemas } \\
\text { relacionados ao trabalho (E) }\end{array}$ & 2,00 \\
\hline Consultar líder de setor (S) & 2,15 \\
\hline Consultar registro “My I get it” (I) & 2,25 \\
\hline Atualizar registros de supervisores (C) & 2,31 \\
\hline Consultar documentos técnicos (I) & 2,38 \\
\hline Realizar benchmarking com outros veículos Ford (I) & 2,39 \\
\hline Realizar benchmarking com veículos da concorrência (I) & 2,41 \\
\hline $\begin{array}{l}\text { Realizar reunião com colegas do BCAD para discutir } \\
\text { problemas técnicos }(S)\end{array}$ & 2,42 \\
\hline $\begin{array}{l}\text { Criar e disponibilizar documento técnico a partir de } \\
\text { outros documentos técnicos (C) }\end{array}$ & 2,45 \\
\hline $\begin{array}{l}\text { Enviar e-mail compartilhando conhecimento próprio } \\
\text { sobre uma técnica ou procedimento }(\mathrm{C})\end{array}$ & 2,54 \\
\hline $\begin{array}{l}\text { Apresentar conhecimento adquirido em cursos } \\
\text { externos à organização (E) }\end{array}$ & 2,60 \\
\hline $\begin{array}{l}\text { Acrescentar comentários adicionais ao registro “My I } \\
\text { get it” (C) }\end{array}$ & 2,88 \\
\hline Publicar resultados de estudos (package, solda, etc.) (E) & 3,00 \\
\hline Consultar tutoriais de software/sistemas (I) & 3,21 \\
\hline $\begin{array}{l}\text { Criar e disponibilizar estudo de benchmarking de } \\
\text { veículos Ford ou da concorrência (C) }\end{array}$ & 3,23 \\
\hline $\begin{array}{l}\text { Publicar projeto Green Belt, Design Rule ou Best } \\
\text { Practice (E) }\end{array}$ & 3,88 \\
\hline
\end{tabular}

$\mathrm{S}=$ Socialização; $\mathrm{E}=$ Externalização; $\mathrm{C}=$ Combinação; $\mathrm{I}$ = Internalização

laços com os colegas. É o caso dos atores $\mathrm{N}_{3}, \mathrm{~N}_{13}$ e N16. Os índices de centralidade média e densidade apontam para uma rede desconectada em alguns pontos. Várias iniciativas podem ser tomadas no sentido de melhorar a integração interna da equipe, como (a) reuniões formais e informais no intuito de promover interação social entre os indivíduos; (b) rotação dos integrantes entre os diferentes subsetores; (c) promoção de um calendário mais intenso de atividades de treinamento, tendo os membros mais experientes da equipe como instrutores; e (d) designação de indivíduos mais experientes para atividades de coaching com aqueles menos experientes. 
Quanto ao relacionamento do BCAD com atores externos, percebem-se ausências importantes. A rede da equipe está isolada em relação a três grupos de atores importantes: o Estúdio de Design, o grupo de Basic Design e o grupo de Gerenciamento de Programas. Nenhum membro dessas equipes parece integrar a rede social informal composta dos relacionamentos dos membros do BCAD com membros externos. Embora a rede social informal do BCAD apresente interessantes e vantajosos relacionamentos espontâneos com outros atores externos, no que se refere aos relacionamentos com os três mencionados grupos, a rede mostrou-se menos eficaz do que a estrutura organizacional formal da companhia. Essa fragilidade parece fácil de ser superada, porque tem como solução o simples reforço de procedimentos formais já previstos. Tal reforço poderá ser viabilizado por workshops entre membros do BCAD e dos três referidos grupos, quando as interfaces de relacionamentos poderão ser discutidas e melhoradas.

Como resultado da análise dos modos de compartilhamento de conhecimentos, percebeu-se uma preferência geral da equipe do BCAD por modos de socialização como meios de captação de conhecimentos e modos de externalização para a transmissão de conhecimentos. De acordo com a classificação de Chang et al. (2011), o BCAD seria uma equipe focada em comunicação (communication-intensive organization). Esse tipo de equipe tem elevado potencial de socialização, demonstra boa abertura para comunicação entre seus integrantes e atribui alta importância às iniciativas de treinamento tipo on-the-job. $\mathrm{Na}$ visão dos mesmos autores, o BCAD teria, também, características de equipe fortemente dotada de analistas simbólicos (symbolic-analyst-dependent organization), em razão do elevado potencial de externalização. Equipes desse tipo têm relativa facilidade para codificar e formalizar procedimentos desenvolvidos pela via da socialização.

Diante dessa constatação, parece importante estimular o uso dos modos baseados em internalização e combinação, de maneira a tentar encontrar-se o equilíbrio entre os quatro modos de conversão do conhecimento dentro da equipe. De acordo com Bhatt (2001), o balanceamento entre os modos de compartilhamento de conhecimento é essencial à efetividade daquele processo. 0 modus operandi para o estabelecimento desse equilíbrio escapa do âmbito deste trabalho, pois requer análise à parte, que pode constar de trabalhos futuros. Com apoio nos estudos de Hansen (1999) e de Sorenson et al. (2006), pode-se adiantar, no entanto, que o ponto de partida para as ações de reforço aos modos de internalização e combinação seria a distinção entre conhecimento complexo (grande volume de dados) e conhecimento simples (menor volume de dados) nas atividades do BCAD. O primeiro tipo de conhecimento teria seu com- partilhamento alavancado pelos sistemas de TI da companhia, enquanto o segundo tipo de conhecimento teria seu compartilhamento incentivado por reforços dos laços das redes sociais. Para uma tentativa de distinção entre conhecimentos complexos e simples, o controle de configuração (configuration control) dos documentos técnicos da companhia forneceria as informações necessárias a essa classificação.

Os autores deste trabalho defrontaram-se com limitações decorrentes do foco restrito em que foram feitas as avaliações, circunscritas a apenas uma equipe do projeto de desenvolvimento do novo EcoSport. Em consequência, a generalização das conclusões e recomendações aqui registradas deve ser vista com prudência. Mesmo reconhecendo as limitações deste estudo, os autores esperam que o trabalho tenha atingido o objetivo inicial de fazer um diagnóstico dos modos e da efetividade do compartilhamento de conhecimentos na equipe do BCAD. Com isso, deu-se um primeiro passo na direção de intervenções no processo de compartilhamento de conhecimento daquela equipe, com vistas a subsidiar o desenvolvimento de produtos vindouros na companhia.

Assume-se, entretanto, que a contribuição maior da investigação está no emprego de instrumentos que têm certo grau de objetividade, para o diagnóstico e proposição de melhorias em uma vertente da gestão caracterizada, em grande medida, pela subjetividade e pela carência de ações concretas, como é o caso da gestão do conhecimento; assim, ao mapear-se a rede social informal da equipe por intermédio dos recursos do software UcinetC, obteve-se o benefício de visualizar a rede e de quantificar as interações entre os seus membros. Esse método contribui para que o compartilhamento de conhecimento seja estudado com o uso de instrumentos mais objetivos e confiáveis.

\section{REFERÊNCIAS}

Andreeva, T, \& Ikhilchik, I. (2011). Applicability of the SECI model of knowledge creation in Russian cultural context: theoretical analysis. Knowledge and Process Management, 18(1), 56-66.

Bhatt, G. D. (2001). Knowledge management in organizations: examining the interaction between technologies, techniques, and people. Journal of Knowledge Management, 5(1), 68-75.

Borgatti, S. P, Everett, M. G, \& Freeman, L. C. (2002). UCINET for Windows: software for social network analysis. Analytic Technologies. Version 6.4 for Windows.

Chang, H.-C, Tsai, M.-T, \& Tsai, C.-L. (2011). Complex organizational knowledge structures for new product development teams. Knowledge-Based Systems, 24(5), 652-661.

Galaskiewicz, J. (1985). Social organization of an urban grants economy: a study of business philanthropy and nonprofit organizations. Orlando, FL, Academic Press. 
Galaskiwicz, J, \& Wasserman, S. (1989). Mimetic processes within an interorganizational field: an empirical test. Administrative Science Quarterly, 34(3), 454-479.

Granovetter, M. (1973). The strength of weak ties. American Journal of Sociology, 78(6), 1360-1380.

Granovetter, M. (1983). The strength of weak ties: a network theory revisited. Sociological theory, 1(1), 201-233.

Hansen, M. T. (1999). The search-transfer problem: the role of weak ties in sharing knowledge across organization subunits. Administrative Science Quarterly, 44(1), 82-111.

Holden, N, \& Glisby, M. (2010). Creating knowledge advantage: the tacit dimensions of international competition and cooperation. Copenhagen: Copenhagen Business School Pr.

Nielsen, A. P. (2006). Understanding dynamic capabilities through knowledge management. Journal of Knowledge Management, 10(4), 5971.

Nonaka, I. and N. Konno (1998). The concept of 'ba': Building a foundation for knowledge creation. California Management Review, 40(3), 40-54.

Nonaka, I, \& Takeuchi, H. (1997). Criação de conhecimento na empresa. Rio de Janeiro: Campus.

Nonaka, I., R. Toyama and N. Konno (2000). SECI, Ba and leadership: a unified model of dynamic knowledge creation. Long Range Planning 33(1), 5-34.
Polanyi, M. (1962). Personal knowledge: towards a post-critical philosophy. London, Routledge \& Kegan Paul Ltd.

Polanyi, M. (1967). The tacit dimension. London, Routledge and Kegan Paul.

Sampaio, R. R, Moraes, M, \& Passos, F. U. (2013). Fluxos de informação e conhecimento no desenvolvimento de produto na indústria automotiva - uma comparação entre as redes formal e informal. Produção Online, 13(1), 208-232.

Sampaio, R. R., C. P. Rosa and H. B. d. B. Pereira (2012). Mapeamento dos fluxos de informação e conhecimento: a governança de TI sob a ótica das redes sociais. Gestão \& Produção 19(2), 377-387.

Sorenson, O, Rivkin, J. W, \& Fleming, L. (2006). Complexity, networks and knowledge flow. Research Policy, 35(7), 994-1017.

Stefanovitz, J. P, \& Nagano, M. S. (2009). Criação de conhecimento na indústria de alta tecnologia: estudo de casos em projetos de diferentes graus de inovação. Revista Gestão \& Produção, 16(2), 245-259.

Wasserman, S, \& Faust, K. (1999). Social network analysis: methods and applications. Cambridge: Cambridge University Press.

Weir, D, \& Hutchings, K. (2005). Cultural embeddedness and contextual constraints: knowledge sharing in Chinese and Arab cultures. Knowledge and Process Management, 12(2), 89-98.

Xu, Y, \& Bernard, A. (2011). Quantifying the value of knowledge within the context of product development. Knowledge-Based Systems, 24(1), 166-175. 